\title{
Vitamin $C$ and aloe vera supplementation protects from chemical hepatocarcinogenesis in the rat
}

\begin{abstract}
The effects of vitamin $\mathrm{C}$ and aloe vera gel extract supplementation on induced hepatocarcinogenesis in male Sprague-Dawley rats (120ï $150 \mathrm{~g}$ ) by diethylnitrosamine (DEN) and 2-acetylaminofluorene (AAF) was investigated. The severity of the carcinogenesis process was determined by measuring J-glutamyl transpeptidase (GGT) and the placental form of glutathione S-transferase (GSTP) histochemically in situ and in plasma and liver fractions. In addition, plasma alkaline phosphatase (ALP) and liver microsomal uridine diphosphate glucuronyl transferase (UDPGT) activity were also determined. Administration of DEN/AAF caused an increase in the surface area and number of enzyme-positive foci (both GGT and GSTP) compared with control. Supplementation of vitamin C or aloe vera gel extract to the cancer-induced rats suppressed this increase significantly $(\mathrm{P}<0.05 ; \mathrm{P}<0.001)$. Increases in liver UDPGT, GGT, and GSTP activities were also observed with cancer induction that were again suppressed with either vitamin $\mathrm{C}$ or aloe vera gel supplementation. Plasma GGT in the DEN/AAF rats were determined monthly for the duration of the experiment and found to be reduced as early as 1 mo with aloe vera gel supplementation and 2 mo with vitamin $\mathrm{C}$ supplementation. In conclusion, vitamin $\mathrm{C}$ and aloe vera gel extract supplementation were found to be able to reduce the severity of chemical hepatocarcinogenesis.
\end{abstract}

Keyword: J-glutamyl transpeptidase; Aloe vera; Chemical hepatocarcinogenesis; Glutathione S-transferase; Vitamin C 